\title{
AN ELEMENTARY PROOF OF A LIMA'S THEOREM FOR SURFACES
}

\author{
F.J. TURIEL
}

Abstract

An elementary proof of the following theorem is given:

THEOREM. Let $M$ be a compact connected surface without boundary. Consider a $C^{\infty}$ action of $\mathbb{R}^{n}$ on $M$. Then, if the Euler-Poincaré characteristic of $M$ is not zero there exits a fixed point.

The proof given here adapts for dimension two the ideas used by P. Molino and the author in [2] and [3]. Moreover we show that the theorem remains true if $\mathrm{R}^{n}$ is replaced by a connected nilpotent Lie group $G$.

In the slightly more general case, dealt with by E.L. Lima, of a surface with boundary, it is sufficient gluing together two copies of this surface in order to obtain a surface without boundary.

\section{Actions of $\mathbf{R}^{n}$}

Let $V$ be the Lie algebra of $R^{n}$. The action of $R^{n}$ induces a Lie algebra homomorphism $v \in V \rightarrow X_{v} \in \mathcal{X}(M)$ called infinitesimal action. We recall that the infinitesimal isotropy of a point $p$ is the set $I(p)=\left\{v \in V / X_{v}(p)=0\right\}$. As $V$ is abelian $l(p)$ depends only on the orbit.

Denote by $\Sigma_{k}$ the set of points $p$ of $M$ whose orbit is $k$-dimensional, i.e. $\operatorname{codim} I(p)=k$.

Suppose $\Sigma_{0}$ empty. We will gradually arrive to a contradiction.

1) Set $C_{2}=\left\{v \in V / X_{v}(p)=0\right.$ for some $\left.p \in \Sigma_{2}\right\}$.

As there are at most countably many 2 -orbits because they are open sets, $C_{2}$ is at most countable union of $(n-2)$-planes of $V$.

2) The map on the grassmamian of $(n-1)$-planes $h: p \in \Sigma_{1} \rightarrow I(p) \in$ $g_{n-1}(V)$ is differentiable, i.e. it can be locally extended to a differentiable map.

Indeed, consider $p \in \Sigma_{1}$ and $u \in V$ such that $X_{u}(p) \neq 0$. We can find a coordinate system $(A, x), p \in A$, such that $X_{u}=\frac{\partial}{\partial x_{1}}$ and that the image of $A$ on $\mathbb{R}^{2}$ is a rectangle. 

map

Let $\left\{v_{1}, \ldots v_{n-1}\right\}$ a basis of $I(p)$. Set $X_{y_{j}}=f_{3} \frac{\partial}{\partial x_{1}}+g_{j} \frac{\partial}{\partial x_{2}}$. We define the

$$
\begin{aligned}
\tilde{h}: A & \longrightarrow g_{n-1}(V) \\
x & \longrightarrow \mathbb{R}\left\{v_{1}-f_{1} u, \ldots v_{n-1}-f_{n-1} u\right\}
\end{aligned}
$$

whose differentiability is clear.

Note that $w \in \tilde{h}(x)$ if and only if $X_{w}(x)$ is proportional to $\frac{\partial}{\partial x_{2}}$. If $x \in$ $A \cap \Sigma_{1}$ this means that $X_{w}(x)=0$ because it is also proportional to $\frac{\partial}{\partial x_{1}}$. Then $\tilde{h}$ is a local extension of $h$.

3) Let $F_{r}\left(\Sigma_{1}\right)$ be the boundary on $M$ of $\Sigma_{1}$. Then $C_{1}=\left\{v \in V / X_{v}(p)=0\right.$ for some $\left.p \in \operatorname{Fr}\left(\Sigma_{1}\right)\right\}=\underset{p \in \operatorname{Fr}\left(\Sigma_{1}\right)}{U} I(p)$ is of the first category (i.e. it is contained in the union of a countable family of closed nowhere dense subsets of $M$ ).

Since $\operatorname{Fr}\left(\Sigma_{1}\right)$ can be covered by a finite family of coordinate systems $(A, x)$ as in 2), it will be sufficient to prove that $\underset{p \in A \cap F r\left(\Sigma_{1}\right)}{U} I(p)$ is of the first category. Let $T$ be a slice of $A$ obtained by doing $x_{1}$ constant. As the isotropy is constant on the orbits:

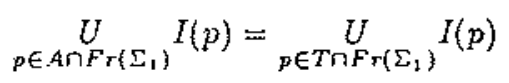

Consider the vector bundle $\pi: E \rightarrow T$, subbundle of $T \times V$, given by the condition $\pi^{-1}(x)=\{x\} \times \tilde{h}(x)$. Set $\varphi:(x, v) \in E \rightarrow v \in V$.

The set $\pi^{-1}\left(\operatorname{T\cap } \operatorname{Fr}\left(\Sigma_{1}\right)\right)$ is of the first category in $E$ because $\operatorname{T\cap } \operatorname{Fr}\left(\Sigma_{1}\right)$ is of the first category in $T$. As $\varphi$ is differentiable and $E$ and $V$ are manifolds of the same dimension, it follows that

$$
\varphi\left(\pi^{-1}\left(T \cap \operatorname{Fr}\left(\Sigma_{1}\right)\right)\right)=\underset{p \in \operatorname{TnFr}\left(\Sigma_{1}\right)}{U} I(p)
$$

is of the first category in $V$.

4) Take now $v \in\left(V-C_{1} \cup C_{2}\right)$. The set $Z\left(X_{v}\right)$ of the zeros of $X_{v}$ is contained in $\Sigma_{1}^{0}$. On the other hand the 1-foliations given by:

(a) $X_{v}$ on $M-Z\left(X_{v}\right)$

(b) the action of $\mathrm{R}^{n}$ on $\stackrel{0}{\Sigma}_{1}$.

agree on $\left(M-Z\left(X_{v}\right)\right) \cap \stackrel{0}{\Sigma}_{1}$. Then $M$ admits an 1 -foliation and $\mathcal{X}(M)=0$, contradiction.

\section{Case of a connected nilpotent Lie group G}

It will be sufficient to adapt the proof of the abelian case. Let $V$ be the Lie algebra of $G$. Since $V$ is nilpotent every subalgebra of codimension one is an 
ideal. Therefore the isotropy is constant over each 1 -orbit and $C_{1}$ will still be of the first category.

Let $B$ be a 2-orbit. Given $p \in B$ there always exists an ideal I of codimension one which contains $I(p)$. As $B$ is an orbit and $I$ an ideal then $I(q) \subset I$ for all $q \in B$. Consequently $C_{2}$ is contained in a finite or countable union of $(n-1)$-planes of $V$. In particular $C_{1} \cup C_{2} \neq V$. The rest is similar.

Example 1. See $P(2, \mathbb{R})$ as the plane $R^{2}$ plus the infinite points. The vector fields on $R^{2}: \frac{\partial}{\partial x_{1}}, \frac{\partial}{\partial x_{2}}$ and $x_{1} \frac{\partial}{\partial x_{2}}$ can be extended, in a natural way, to $P(2, \mathbb{R})$ because they are affine. These vector fields generate an action of a 3-dimensional nilpotent group on $P(2, R)$, whose orbits are $R^{2}$; the set of all points of infinity except the vertical one (i.e. the point associated to the vertical direction); and the infinite vertical point, which is the only fixed point.

Example 2. Tare now $\frac{\partial}{\partial x_{1}}, \frac{\partial}{\partial x_{2}}$ and $-x_{2} \frac{\partial}{\partial x_{1}}+x_{1} \frac{\partial}{\partial x_{2}}$. One obtains an action of a 3-dimensional solvable group with no fixed point. Their orbits are $R^{2}$ and the set of the infinite points.

See [1] for a 2-dimensional example with no fixed point.

\section{References}

1. E.L. LIMA, Common singularities of commuting vector fields on 2-manifolds, Comment. Math. Helvet. 39 (1964), 97-110.

2. P. Molino, F.J. TURIEL, Une observation sur les actions de $R^{p}$ sur les variétés compactes de caractéristique non nulle, Comment. Math. Helvet. 61 (1986), 370-375.

3. P. Molino, F.J. TURIEL, Dimension des orbites d'une action de $\mathbf{R}^{p}$ sur une variété compacte, Comment. Math. Helvet. 63 (1988), 253-258.

\footnotetext{
Sección de Matemáticas

Facultad de Ciencias, Ap. 59

29080 Malaga. SPAIN
}

Rebut el 26 de Gener de 1989 